\title{
Transcriptome analysis identifies genes involved in the somatic embryogenesis of Eucalyptus
}

\author{
Yufei Xiao ${ }^{\dagger}$, Junji Li ${ }^{\dagger}$, Ye Zhang, Xiaoning Zhang, Hailong Liu, Zihai Qin and Bowen Chen ${ }^{*}$ (D)
}

\begin{abstract}
Background: Eucalyptus, a highly diverse genus of the Myrtaceae family, is the most widely planted hardwood in the world due to its increasing importance for fiber and energy. Somatic embryogenesis (SE) is one large-scale method to provide commercial use of the vegetative propagation of Eucalyptus and dedifferentiation is a key step for plant cells to become meristematic. However, little is known about the molecular changes during the Eucalyptus SE.

Results: We compared the transcriptome profiles of the differentiated and dedifferentiated tissues of two Eucalyptus species - E. camaldulensis (high embryogenetic potential) and E. grandis $x$ urophylla (low embryogenetic potential). Initially, we identified 18,777 to 20,240 genes in all samples. Compared to the differentiated tissues, we identified 9229 and 8989 differentially expressed genes (DEGs) in the dedifferentiated tissues of E. camaldulensis and E. grandis $x$ urophylla, respectively, and 2687 up-regulated and 2581 down-regulated genes shared. Next, we identified 2003 upregulated and 1958 down-regulated genes only in E. camaldulensis, including 6 somatic embryogenesis receptor kinase, 17 ethylene, 12 auxin, 83 ribosomal protein, 28 zinc finger protein, 10 heat shock protein, 9 histone, 122 cell wall related and 98 transcription factor genes. Genes from other families like ABA, arabinogalactan protein and late embryogenesis abundant protein were also found to be specifically dysregulated in the dedifferentiation process of $E$. camaldulensis. Further, we identified 48,447 variants (SNPs and small indels) specific to E. camaldulensis, including 13, 434 exonic variants from 4723 genes (e.g., annexin, GN, ARF and AP2-like ethylene-responsive transcription factor). qRTPCR was used to confirm the gene expression patterns in both E. camaldulensis and E. grandis $x$ urophylla.

Conclusions: This is the first time to study the somatic embryogenesis of Eucalyptus using transcriptome sequencing. It will improve our understanding of the molecular mechanisms of somatic embryogenesis and dedifferentiation in Eucalyptus. Our results provide a valuable resource for future studies in the field of Eucalyptus and will benefit the Eucalyptus breeding program.
\end{abstract}

Keywords: Eucalyptus, Vegetative propagation, Somatic embryogenesis, Dedifferentiation, Transcriptome, Callus

\footnotetext{
* Correspondence: bwchen_gfri@163.com

${ }^{\dagger}$ Yufei Xiao and Junji Li contributed equally to this work.

Guangxi Key Laboratory of Superior Timber Trees Resource Cultivation, Guangxi Forestry Research Institute, 23 Yongwu Road, Nanning 530002, Guangxi, China
} 


\section{Background}

Eucalyptus is a highly diverse genus of the Myrtaceae family and is widely planted across the world for its increasing importance for timber and pulp [1]. Natural regeneration of Eucalyptus mainly relies on seeds, however, their breeding is always a slow process due to the length of the juvenile phase [1]. Thus, vegetative propagation becomes an alternative option for Eucalyptus to breed and to gain new characteristics. Vegetative propagation consists of methods of both micropropagation (e.g., air layering, grafting, rooting of cuttings) and micropropagation using in vitro tissue culture techniques, including adventitious budding, axillary shoot tips, somatic embryogenesis (SE) [2]. Among them, in vitro tissue culture-induced $\mathrm{SE}$, in which whole fertile plants are regenerated under proper culture conditions, is widely used to propagate selected genotypes for commercial purposes because it can provide large-scale regenerations for plants with relative lower cost [2].

In forest trees, $\mathrm{SE}$ is a complex process involving many factors in different steps [2]. The first step is the callus induction in which differentiated somatic cells (e.g., seed, leaf, stem) acquire embryogenetic competence with or without a dedifferentiation step [3]. In Eucalyptus, Ouyang et al. reported the SE from the callus of seedlings for the first time in 1980 [4]. Pinto reviewed the status and future perspectives of SE in Eucalyptus [5]. Some tissues (e.g., hypocotyls, cotyledons, leaves, shoots) from both young seedlings and old trees have been reported for successful induction of callus in multiple Eucalyptus species, such as E. botryoides, E. dunnii, E. grandis, E. globulus and E. rudis [5]. Including the culture medium, some other factors have been reported to affect the SE induction, such as the addition of antioxidants, the carbon source, the effect of genotype and the morphogenic pathway [5]. It is clear that the capacity of SE in Eucalyptus species varies, however, our knowledge about the genomic and transcriptomic information controlling the SE and vegetative propagation is still poor.

Some studies have reported genetics associated with the capacity of vegetative propagation in Eucalyptus. For example, Grattapaglia used a pseudo-testcross strategy and RAPD markers to study the quantitative trait loci (QTLs) controlling vegetative propagation in E. grandis and E. urophylla [6]. Marques identified some QTLs related to adventitious rooting, sprouting ability and the stability of adventitious rooting [7]. Thumma identified some QTLs related to the wood quality in Eucalyptus nitens [8]. Ohtani summarized the impacts of the regulation of RNA metabolism for in vitro dedifferentiation of plant cells, such as rRNA biosynthesis, pre-mRNA splicing, and miRNA-based RNA decay [9]. In addition, gene regulation in SE has been studied in plants, such as Arabidopsis [10], alfalfa [11], camphor tree [12], carrot [13], cotton [14-16], orange [17], potato [16] and soybean [18-20]. Many genes have been reported to function in SE, such as auxin response factor19 (ARF19), somatic embryogenesis receptor-like kinase (SERK), leafy cotyledon (LEC), baby boom (BBM), and wuschel (WUS) [21, 22]. However, it is still unknown about the gene changes and gene regulations in the SE and dedifferentiation of Eucalyptus.

In the present study, we analyzed the transcriptome profiles and gene variants in the dedifferentiation process of two Eucalyptus species - E. camaldulensis (high embryogenetic potential) and E. grandis $x$ urophylla (low embryogenetic potential). Some genes were identified to relate to the SE and dedifferentiation of Eucalyptus, such as auxin related genes, embryogenesis related genes, ethylene related genes, ribosomal protein (RP) genes, zinc finger protein (ZFP) genes, histone genes, heat shock protein (HSP) genes and various transcription factors (TFs). Further, we identified functional variants in these two Eucalyptus species that might control the callus induction and development. This is the first time to report the SE transcriptomes in Eucalyptus. It will improve our understanding about the molecular mechanisms in the SE of Eucalyptus. Further, the output of this study will provide a valuable resource for future studies and will benefit the research in this field, especially the breeding program of Eucalyptus.

\section{Results \\ Morphological characterization of somatic callus}

To study the somatic embryogenesis of Eucalyptus, the stem (differentiated) segments of both E. camaldulensis (A1) and E. grandis $x$ urophylla (A2) were tissue-culture induced to callus (B1 for E. camaldulensis and B2 for E. grandis $x$ urophylla). In these two Eucalyptus species, the morphologies (Fig. 1a) of the tissue culture induced callus were similar and their growth curves (Fig. 1b) showed Sshaped during dedifferentiation. The weight and appearance of the stem had no significant change during the first 2 days of induction. After 3 to 6 days induction, the two ends of the stem started to be induced to callus, which showed that both ends turned pale yellow, expanded and thickened like dumbbell (Fig. 1a). Then, it fell into the rapid growth period of 7 to 12 days induction. The dedifferentiated callus gradually extended from both ends to the middle and the whole stem was completely dedifferentiated into pale yellow and moist callus. After 13 days of induction, it entered into the slow growth stage. The callus which had been cultured in the induction medium for 10 days were used for transcriptome sequencing.

\section{Transcriptome sequencing and gene expression profiles}

Next, we analyzed the transcriptome profiles of differentiated and dedifferentiated tissues of E. camaldulensis and E. grandis. Table 1 showed an overview of the 


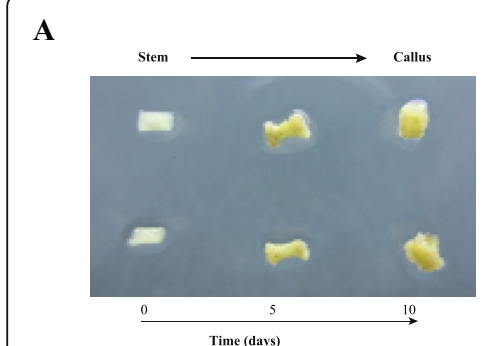

D

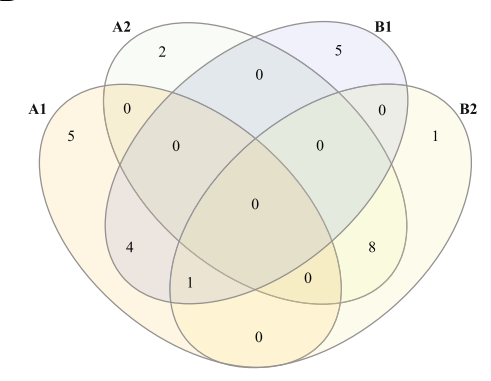

B

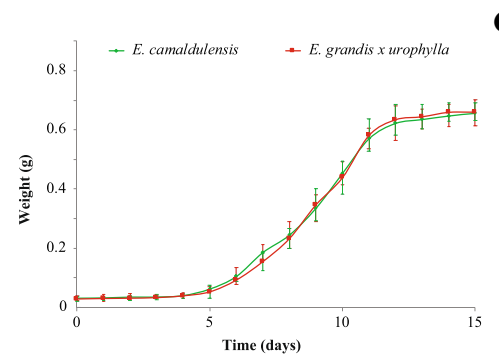

$\mathbf{E}$

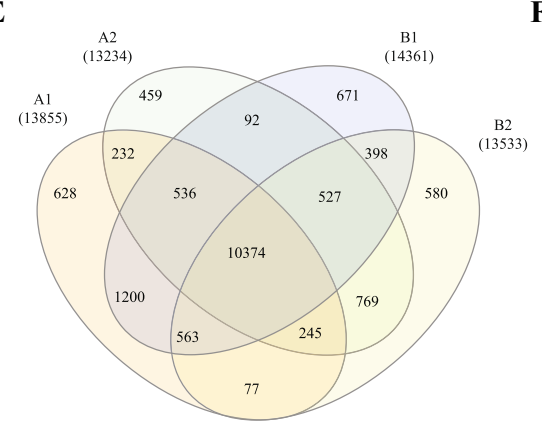

C

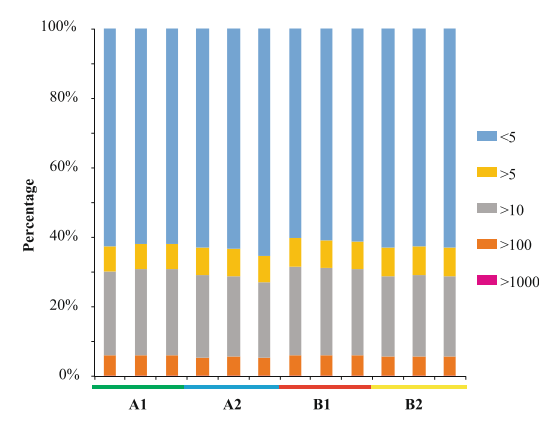

$\mathbf{F}$

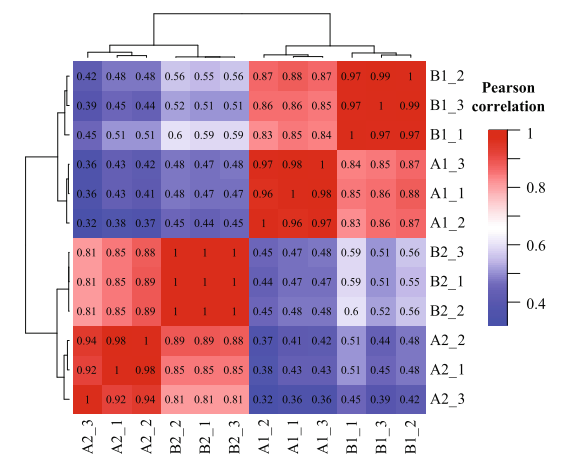

Fig. 1 Morphological characterization of somatic callus and overview of the transcriptome sequencing. a Morphological characterization of the dedifferentiation from stem to callus. Upper panel: E. camaldulensis; lower panel: E. grandis x urophylla. $\mathbf{b}$ Growth curves of the callus tissue of $E$. camaldulensis and E. grandis $x$ urophylla. $\mathbf{c}$ Number of genes identified in the stem and callus of E. camaldulensis (A1, A2) and E. grandis $x$ urophylla (B1, B2). $\mathbf{d}$ Venn diagram of top 10 highly expressed genes in all samples. e Venn diagram of genes (TPM > 1) identified in all samples. $\mathbf{f}$ Heat map of sample correlation based on the gene expression profiles. Color bar represents the values of Pearson correlation

transcriptome sequencing and the numbers of genes identified in each sample. Initially, we obtained 528 million reads for all 12 samples (in triplicates, $n=3$ ) with an average of 44 million reads. Then, the reads were aligned to the E. grandis genome and it showed that 64.66 to $74.84 \%$ of the total reads were mapped. Out of the total 36,349 E. grandis genes, we identified 18,777 to 20,240 genes with TPM $>1$ in the differentiated and dedifferentiated tissues of Eucalyptus (Additional file 1). When we filtered genes using $\mathrm{TPM}<5$, only 12,650 to 14,464 genes were identified. Figure 1c showed that more than $60 \%$ of the genes were lowly expressed $(\mathrm{TPM}<5)$ and only 0.165 to $0.272 \%$ of the Eucalyptus genes were expressed higher than 1000 TPM. Interestingly, highly expressed genes varied in differentiated and dedifferentiated tissues of Eucalyptus. Figure 1d showed the comparison of top 10 highly expressed genes in all samples according to the average TPM. Dedifferentiated tissues

Table 1 Overview of transcriptome sequencing and gene expression profiles

\begin{tabular}{|c|c|c|c|c|c|}
\hline Sample name & Total reads & Mapped reads & Percentage & Genes (TPM > 1) & Genes (TPM > 5) \\
\hline A1_1 & $43,673,352$ & $32,135,161$ & $73.58 \%$ & 18,777 & 13,561 \\
\hline A1_2 & $43,658,680$ & $32,673,328$ & $74.84 \%$ & 19,024 & 13,855 \\
\hline A1_3 & $43,743,786$ & $30,350,588$ & $69.38 \%$ & 19,115 & 13,858 \\
\hline$A 2 \_1$ & $41,999,356$ & $27,550,545$ & $65.60 \%$ & 18,950 & 13,435 \\
\hline$A 2 \_2$ & $43,718,576$ & $28,266,987$ & $64.66 \%$ & 19,094 & 13,360 \\
\hline$A 2 \_3$ & $41,978,434$ & $27,287,763$ & $65.00 \%$ & 18,406 & 12,650 \\
\hline B1_1 & $45,524,984$ & $32,704,591$ & $71.84 \%$ & 20,240 & 14,464 \\
\hline B1_2 & $43,753,980$ & $31,740,739$ & $72.54 \%$ & 20,186 & 14,274 \\
\hline B1_3 & $45,492,018$ & $32,752,661$ & $72.00 \%$ & 19,942 & 14,147 \\
\hline B2_1 & $43,661,620$ & $32,556,137$ & $74.56 \%$ & 19,322 & 13,518 \\
\hline B2_2 & $47,151,700$ & $35,051,250$ & $74.34 \%$ & 19,338 & 13,567 \\
\hline B2_3 & $43,651,908$ & $32,069,801$ & $73.47 \%$ & 19,276 & 13,536 \\
\hline
\end{tabular}


of both E. camaldulensis and E. grandis $x$ urophylla shared 8 highly expressed genes including Eucgr.H01085 (ethylene-responsive transcription factor ERF071), Eucgr.G03106 (wound-induced protein 1) and Eucgr.F00114 (zinc finger protein ZAT10). We next compared the genes identified in all the samples (TPM > 1) and found 10,374 genes shared (Fig. 1e). In addition, 628, 459, 671 and 580 genes were specifically identified in A1, A2, B1 and B2, respectively. Based on the gene expression we analyzed the correlation between samples. It was revealed that replicates were performed well and that differentiated tissues were distinguishable from dedifferentiated tissues in E. camaldulensis and E. grandis $x$ urophylla (Fig. 1f).

\section{DEGs in E. camaldulensis}

To study the possible functional genes/pathways in the SE of Eucalyptus, we first identified DEGs in the dedifferentiated tissue of E. camaldulensis compared to the differentiated tissue. Using edgeR we identified 4690 up-regulated and 4539 down-regulated genes (Fig. 2a, Additional file 2). It showed that Eucgr.H02264 (probable indole-3-acetic acid-amido synthetase GH3.1), Eucgr.D02625 (phosphoribulokinase, chloroplastic), Eucgr.J03055 (hypothetical protein), Eucgr.H02600 (protein SRG1) and Eucgr.B03016 (LOB domain-containing protein 40) were the top 5 upregulated genes while Eucgr.J00794 (DNA-damage-repair/ toleration protein DRT100), Eucgr.I02271 (endochitinase), Eucgr.A01080 (glycine-rich RNA-binding protein), Eucgr.D00703 (beta-galactosidase 8) and Eucgr.A01881 (trans-resveratrol di-O-methyltransferase) were the top 5 down-regulated genes, according to the FDR (false discovery rate) values (Additional file 2). We next analyzed the KEGG pathways enriched by the DEGs and found that "plant hormone signal transduction" (ko0475), "RNA transport" (ko03013) and "carbon metabolism" (ko01200) were the top 3 significant pathways. GO enrichment analysis (Fig. 2b) showed that together with the GO terms enriched by more than $10 \%$ of the DEGs, "reproduction" and "reproduction process" were found with interest.

Then, we analyzed some gene groups might be involved in the dedifferentiation process of E. camaldulensis. Initially, 27 up-regulated and 22 down-regulated genes were found to associate with ethylene (Table 2), including 7 AP2-like ethylene-responsive TFs, 41 ethyleneresponsive TFs and 1 ethylene response sensor (Additional file 2). In addition, 41 DEGs encoding auxin related products were found in the callus of $E$. camaldulensis compared to the stem (Table 2), including 7 auxin response factors, 3 auxin-binding proteins, 2 auxin-induced in root clusters proteins and 16 auxin-responsive proteins (Additional file 2). Furthermore, we found 111, 79, 36, 39 and 272 DEGs (Table 2) encoding RP, ZFP, HSP, histone and TF, respectively, might be involved in the dedifferentiation process of E. camaldulensis. Notably, 8 up-regulated and 3 down- regulated embryogenesis related genes were identified in the callus of E. camaldulensis compared to the stem, such as late embryogenesis abundant protein, somatic embryogenesis receptor kinas 1 and embryogenesisassociated protein EMB8 (Additional file 2). Further, based on the GO annotation we identified 274 DEGs (154 up-regulated and 120 down-regulated) related to cell wall (Table 2). Details of these cell wall related DEGs in different categories, such as "GO:0009505 plant-type cell wall" and "GO:0009834 plant-type secondary cell wall biogenesis", can be accessed in the additional file 3 . Among them, 9 DEGs encoding expansin were upregulated and 1 gene encoding PME53 (probable pectinesterase 53) were down-regulated.

\section{DEGs in E. grandis $\mathrm{x}$ urophylla}

We next identified 4200 up-regulated and 4708 downregulated genes in the dedifferentiated tissue of $E$. grandis $x$ urophylla compared to the differentiated tissue (Fig. 2c). According to the FDR, top 5 DEGs include Eucgr.B03016 (LOB domain-containing protein 40), Eucgr.H02264 (probable indole-3-acetic acid-amido synthetase GH3.1), Eucgr.I02271 (endochitinase), Eucgr.L02894 and Eucgr.L02534 (Additional file 2). Notably, pathway analysis identified "Plant hormone signal transduction" (ko04075) significantly enriched by 260 DEGs (q-value: 8.36E-14), which indicates that these DEGs may play key roles in the SE of E. grandis $x$ urophylla. GO analysis (Fig. 2d) showed that most of the GO terms involved by the DEGs were shared by E. grandis $x$ urophylla and E. camaldulensis but some might be specific to E. grandis $x$ urophylla, such as biological adhesion, virion and nucleoid.

Table 2 showed the numbers of DEGs from the seven gene groups identified in the dedifferentiation process of E. grandis $x$ urophylla. Interestingly, we found more DEGs (41 up-regulated and 8 down-regulated) encoding ribosomal proteins in E. grandis $x$ urophylla than E. camaldulensis, especially some RP genes which were up-regulated in E. grandis $x$ urophylla but down-regulated in E. camaldulensis. In addition, compared to E. camaldulensis more down-regulated ethylene related genes and more upregulated HSP and histone genes were found in E. grandis $x$ urophylla (Table 2). Figure 2e showed an overview of all the DEGs identified in the dedifferentiated tissues compared to the differentiated tissues in both E. camaldulensis and E. grandis $x$ urophylla. It showed that some DEGs were specifically identified in E. camaldulensis or $E$. grandis $x$ urophylla, which might relate to the regenerative ability of Eucalyptus.

\section{Regenerative ability associated genes}

We next compared the DEGs identified in the dedifferentiation process of $E$. camaldulensis and E. grandis $x$ urophylla. It showed in the upper panel of Fig. 3a that 


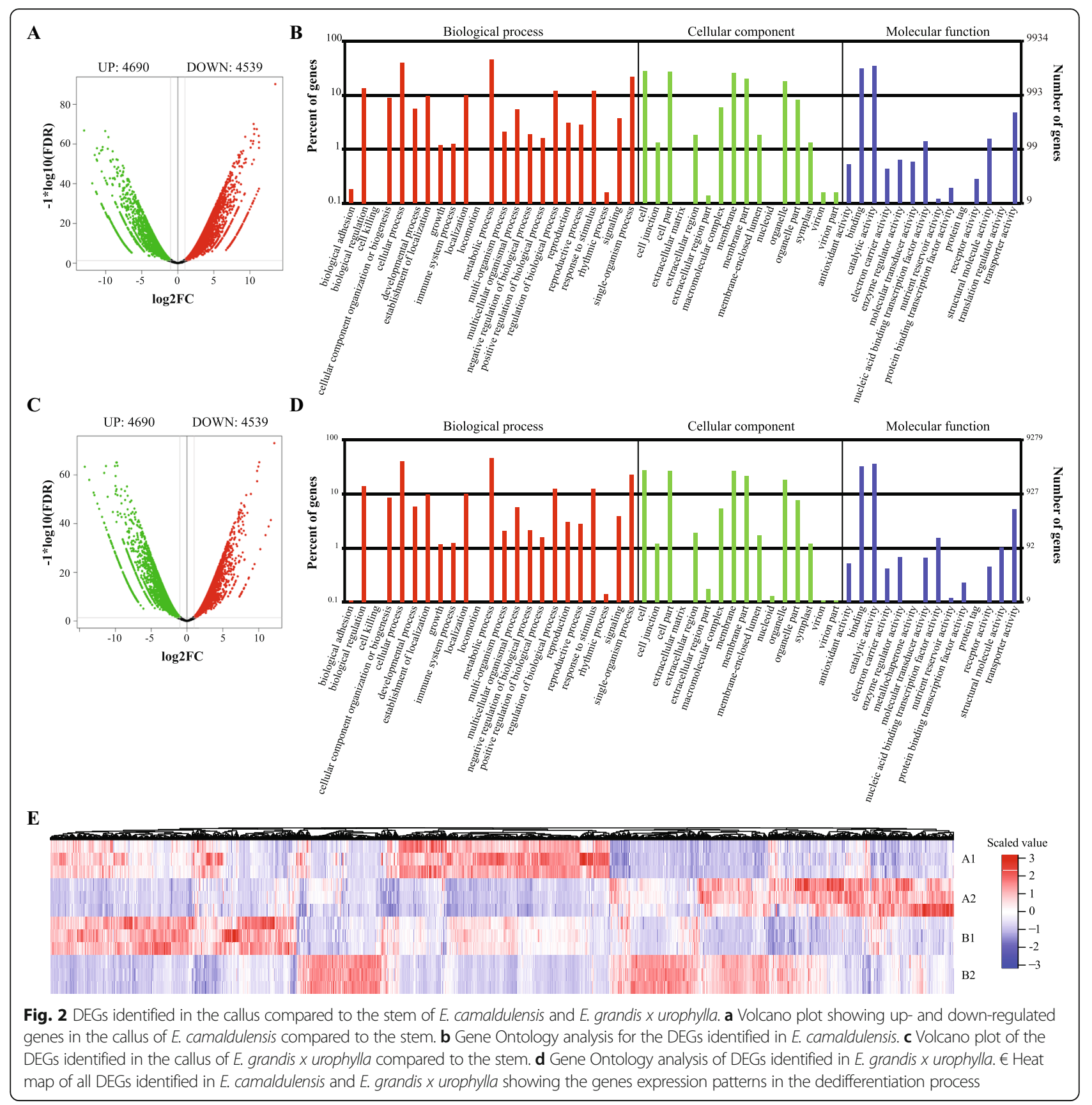

they shared 2687 up-regulated genes in the dedifferentiated tissue compared to differentiated tissue, including Eucgr.H01085 (ethylene-responsive transcription factor ERF071), Eucgr.A01538 (fructose-bisphosphate aldolase 6, cytosolic), Eucgr.G03106 (wound-induced protein 1), Eucgr.K02614 (NDR1/HIN1-Like protein 3), Eucgr.F00114 (zinc finger protein ZAT10) and Eucgr.H03082 (early nodulin-75) (Additional file 2). There were 2003 and 1513 up-regulated genes specifically identified in the callus of E. camaldulensis and E. grandis $x$ urophylla, respectively (upper panel of Fig. 3a). Then, we compared to down-regulated genes and found that they shared 2581 genes (lower panel of Fig. 3a), including Eucgr.J00025 (heat shock cognate $70 \mathrm{kDa}$ protein 2), Eucgr.B01596 (probable xyloglucan endotransglucosylase/hydrolase protein 23), Eucgr.A01080 (glycine-rich RNA-binding protein), Eucgr.F00590 (snakin-2) and Eucgr.H03983 (major allergen Pru ar 1) (Additional file 2). A total of 1958 and 2127 down-regulated genes were specifically identified in E. camaldulensis and E. grandis $x$ urophylla, respectively (lower panel of Fig. 3a). 
Table 2 Genes differentially expressed in dedifferentiated tissues compared to differentiated tissues in Eucalyptus

\begin{tabular}{|c|c|c|c|c|}
\hline Gene family & E. camaldulensis & E. grandis $x$ urophylla & Shared & E. camaldulensis only \\
\hline Ethylene related & $27 / 22^{a}$ & $16 / 37$ & $15 / 17$ & $12 / 5$ \\
\hline Auxin & $25 / 16$ & $18 / 18$ & $15 / 14$ & $10 / 2$ \\
\hline Ribosomal protein & $15 / 86$ & $41 / 8$ & $12 / 6$ & $3 / 80$ \\
\hline Zinc finger protein & $35 / 44$ & $38 / 38$ & $28 / 23$ & $7 / 21$ \\
\hline Heat shock protein & $23 / 13$ & $29 / 6$ & $21 / 5$ & $2 / 8$ \\
\hline Histone & $27 / 12$ & $33 / 11$ & $24 / 6$ & $3 / 6$ \\
\hline TF & $155 / 117$ & $128 / 143$ & $95 / 79$ & $60 / 38$ \\
\hline ABA & $3 / 5$ & $5 / 4$ & $3 / 2$ & $0 / 3$ \\
\hline AGP & $5 / 3$ & $4 / 6$ & $2 / 3$ & $3 / 0$ \\
\hline Embryogenesis & $8 / 3$ & $5 / 3$ & $3 / 2$ & $5 / 1$ \\
\hline Cell wall & $154 / 120$ & $131 / 120$ & $86 / 66$ & $68 / 54$ \\
\hline
\end{tabular}

${ }^{\mathrm{a}}$ Molecule and denominator represent the numbers of up- and down-regulated genes, respectively

Interestingly, we identified 6, 17, 12, 83, 28, 10, 9, 122 and 98 DEGs related to the embryogenesis, ethylene, auxin, RP, ZFP, HSP, histone, cell wall and TF, respectively, only in the dedifferentiation process of E. camaldulensis (Table 2, Additional file 2). The 6 embryogenesis related genes include 5 up-regulated genes (2 SE receptor kinase, 3 LEA) and 1 downregulated gene encoding embryogenesis-associated protein EMB8. Among the 17 DEGs encoding auxin related products (Fig. $3 \mathrm{~b}$ ) specifically identified in E. camaldulensis, Eucgr.K02992 (auxin transporter-like protein 4) and Eucgr.C02984 (auxin-responsive protein IAA26) were down-regulated and Eucgr.H03171 (auxin-induced protein 22D) was up-regulated in E. camaldulensis but down-regulated in E. grandis $x$ urophylla (Additional file 2). All the DEGs related to ethylene and specifically identified in the callus of E. camaldulensis were found to encode ER TFs, and 7 had reverse regulation in $E$. camaldulensis and E. grandis $x$ urophylla (Fig. 3c). Interestingly, heat maps (Fig. 3d and e) showed that most of the E. camaldulensis specific DEGs encoding HSP and $\mathrm{RP}$ were down-regulated in the dedifferentiated tissue compared to the differentiated tissue. Although some of the DEGs encoding histone were more or less changed in E. grandis $x$ urophylla, the difference of their expression was not significant as that in E. camaldulensis (Fig. 3f). Importantly, we found another 4 TF subfamilies including ASIL2, bHLH, MYB and WRKY were dysregulated only in E. camaldulensis (Fig. 3g). Further, the expression of MYB and WRKY TF genes were elevated during the dedifferentiation process of E. camaldulensis. The cell wall related DEGs specific to E. camaldulensis were involved in multiple process, such as "GO: 0005199 structural constituent of cell wall", "GO: 0009664 plant-type cell wall organization" and "GO: 0009832 plant-type cell wall biogenesis" (Additional file 3). It is interesting that Eucgr.F01000 (formin-like protein $5, \log 2 \mathrm{FC}=1.65, p=5.67 \mathrm{E}-05$ ) were the only DEG involved in the "GO:0005199 structural constituent of cell wall". In addition, some other gene families were identified to be specifically differentially expressed in the SE of E. camaldulensis (Table 2, Additional file 3), such as 3 ABA related genes, 3 arabinogalactan protein genes, $4 \mathrm{ABC}$ transporter genes and 21 abscisic stressripening protein genes.

\section{qRT-PCR validation}

We used qRT-PCR to confirm the expression patterns of DEGs in the dedifferentiation process of $E$. camaldulensis and $E$. grandis $x$ urophylla. We randomly selected 9 genes (Eucgr.A00971, Eucgr.A01091, Eucgr.B03715, Eucgr.C03048, Eucgr.D01811, Eucgr.F00490, Eucgr.F01164, Eucgr.H03077 and Eucgr.K01605) for the qRT-PCR experiment and the $\mathrm{H} 2 \mathrm{~B}$ gene was used as the internal control. The primer sequences for all these genes can be accessed in the Additional file 4. Each gene was replicated three time in every sample, so we performed 9 reactions in total for the differentiated and dedifferentiated tissues. Log2 fold change $(\log 2 \mathrm{FC})$ and $\log 2$ relative normalized expression $(\log 2 \mathrm{RNE})$ were used to present the gene changes detected by transcriptome sequencing and qRT-PCR, respectively. Overall, $14(77.8 \%)$ out of 18 events were agreed by both qRT-PCR and deep sequencing (Fig. 4). Interestingly, the upregulation of SE marker gene SERK1 was detected by qRTPCR in both Eucalyptus species while transcriptome sequencing only detected its up-regulation in E. camaldulensis. However, other DEGs encoding SERK1 were identified with up-regulation in E. grandis $x$ urophylla (Additional file 2). It is notable that the increase of WRKY TF (Eucgr.D01811) and the decrease of RP gene (Eucgr.A00971) in E. camaldulensis were confirmed by qRT-PCR. The dysregulation of Eucgr.B03715, Eucgr.C03048 and Eucgr.F00490 in E. grandis $x$ urophylla were also confirmed. High agreement of gene expression patterns in transcriptome sequencing and qRT- 


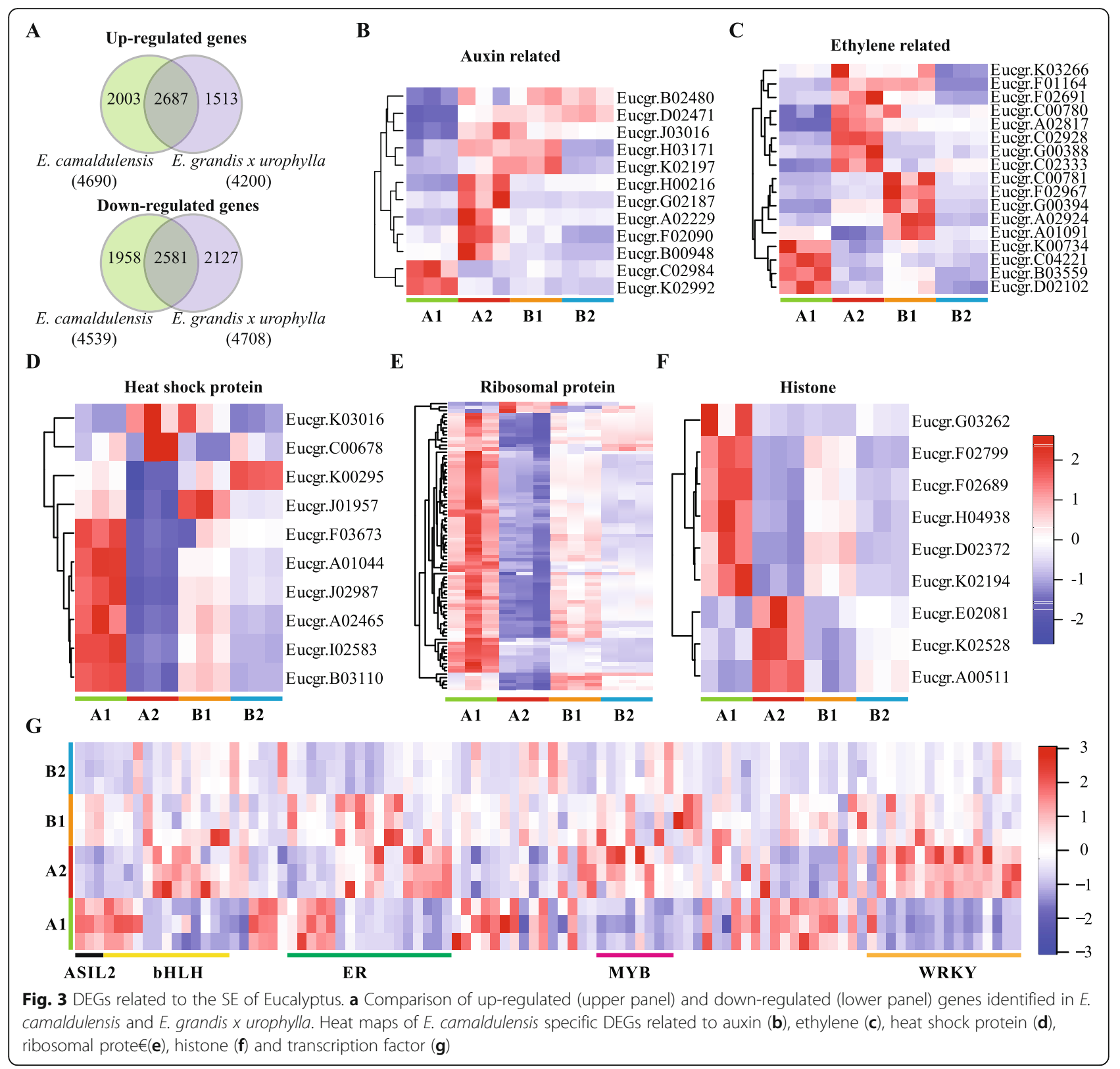

PCR indicate that the genes identified in this study might be associated with the regenerative ability and the SE of Eucalyptus, which requires future experiments to be explored.

\section{SNPs and indels}

We next identified gene variants (e.g., SNPs and small indels) in E. camaldulensis and E. grandis $x$ urophylla using the transcriptome sequencing data. Initially, we obtained 97,504 and 75,582 variants in the differentiated and dedifferentiated tissues of E. camaldulensis, respectively (Table 3 ). After the variants supported by $<100$ reads were filtered, we identified 97,974 variants for $E$. camaldulensis. Likewise, 72,208 and 66,311 variants were found in the differentiated and dedifferentiated tissues of E. grandis $x$ urophylla, respectively, and they produced 78,977 variants after filtering variants with low supportive reads (Table 3). Comparison showed 49,527 variants shared by $E$. camaldulensis and E. grandis $x$ urophylla, and 48,447 variants were specifically identified in E. camaldulensis. Then, we annotated the $E$. camaldulensis specific variants using ANNOVAR and found that 13,434 variants were functional, such as nonsynonymous, frameshift insertion, frameshift deletion, stop gain and stop loss variants (Table 3, Additional file 5). Interestingly, these 13,434 variants were derived from 4723 Eucalyptus genes, such as annexin (Eucgr.F02423, Eucgr.H00564), ARF guanine-nucleotide exchange factor GNOM (Eucgr.B03196), AP2-like ER 

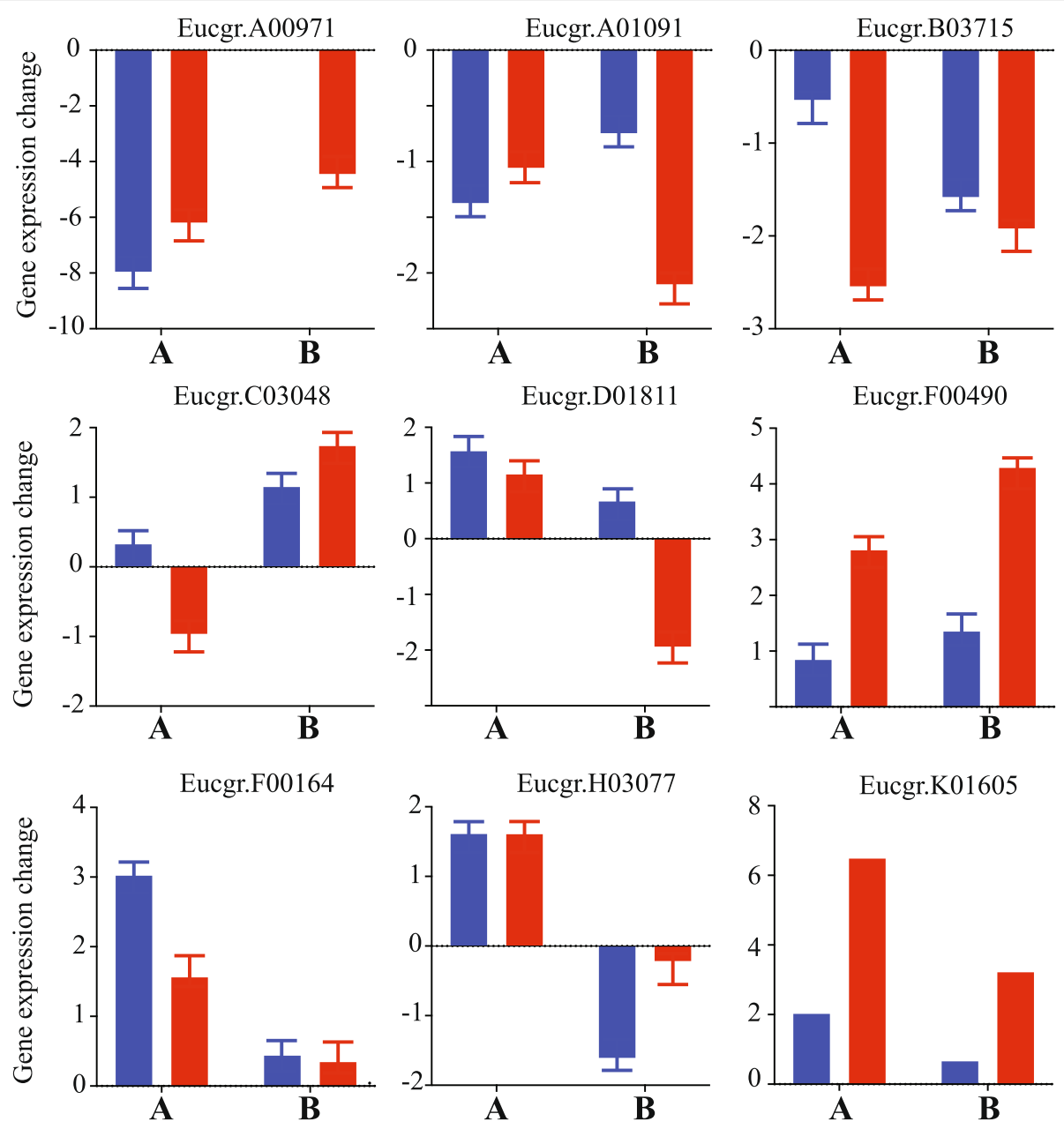

$\log 2 \mathrm{FC}$

$\log 2 \mathrm{RNE} \quad \mathrm{A}:$ E. camaldulensis

B: E. grandis $x$ urophylla

Fig. 4 qRT-PCR validation. Log2FC and log2RNE represent the gene expression changes identified by the transcriptome sequencing and qRT-PCR, respectively

TF (Eucgr.I00278, Eucgr.J02113), auxin response factors (Eucgr.G00076, Eucgr.C02178, Eucgr.C03293, Eucgr.J00923, Eucgr.F02090, Eucgr.D00264, Eucgr.E00888) and wallassociated receptor kinas-like (Eucgr.I01022). KEGG pathway analysis showed that 85 pathways including "longevity

Table 3 Gene variants identified in E. camaldulensis and E. grandis $x$ urophylla

\begin{tabular}{llllll}
\hline & \multicolumn{2}{l}{ E. camaldulensis } & & \multicolumn{2}{l}{ E. grandis $\boldsymbol{x}$ urophylla } \\
\cline { 2 - 3 } \cline { 5 - 6 } & A1 & A2 & & B1 & B2 \\
\hline Variants & 97,504 & 75,582 & & 72,208 & 66,311 \\
DP $^{\mathrm{a}}>100, n>3$ & 97,974 & & 78,977 & \\
Common & 49,527 & & & \\
Specific & 48,447 & & 29,450 & \\
Functional variants & 13,434 & & 7684 & \\
Involved genes & 4723 & & 3609 & \\
\hline
\end{tabular}

${ }^{\mathrm{a}} D P$ read depth regulating pathway" (ko04211) and "Plant hormone signal transduction" (ko04075) were enriched by the of the $E$. camaldulensis specific mutated genes (Additional file 6).

\section{Discussions}

SE has become an efficient way for the propagation and SERK genes have been reported to play a key role during the SE of many plants, such as Arabidopsis [23], cacao [24], rice [25], sunflower [26], maize [27], grape [28] and pineapple [29]. In Arabidopsis, SERK1 was highly expressed during the formation of embryogenic cells and can be detectable in all cells of the developing embryo during early SE up to the formation of the heart stage [23]. SERK2 is significantly increased in the embryogenic callus and the maturation stage compared to nonembryogenic callus [30]. In the present study we identified the up-regulation of some SERK genes in the dedifferentiated tissue compared to differentiated tissue in both E. camaldulensis and E. grandis $x$ urophylla 
(Additional file 2). Further, we found that 2 SERK gene members were specifically up-regulated in the dedifferentiation of $E$. camaldulensis. These results suggested that different members of the SERK gene family may have diverse functions. Singh and Khurana also observed the different expression patterns of SERK genes in wheat and reported that SERK genes were differentially expressed in response to different plant growth regulators [31]. For example, SERK2 and SERK3 can elicit the auxin-specific responses while SERK1 and SERK5 may be mediated by the signaling pathway of brassinosteroids. The functions of SERK genes in the SE of Eucalyptus require further experiments to be explored.

Méndez-Hernández reviewed the interactions between different plant growth regulators, mainly auxins, cytokinin, ethylene and abscisic acid (ABA), during the induction of SE [32]. Although how the cells initiate embryo formation is not clear, an irregular distribution of auxins must be established to initiate embryo formation. In the present study, three genes (Eucgr.B00948, Eucgr.G02187, Eucgr.A02229) encoding auxin efflux carrier were upregulated only in the dedifferentiation process of $E$. camaldulensis (Table 2, Additional file 3). In addition, three genes encoding auxin response factors were specifically up-regulated in E. camaldulensis. We also identified DEGs related to other plant hormones, like late embryogenesis abundant protein (LEA) and abscisic acid (ABA). For example, twelve ABA related genes were dysregulated, including seven $A B A$ receptor and five 8 '-hydroxylase genes (Additional file 3). Among them, one ABA receptor and two ABA 8'-hydroxylase genes were specifically down-regulated in the dedifferentiated tissue of E. camaldulensis. Chen et al, reported that the ABA transcripts peaked at $8 \mathrm{~h}$ after $\mathrm{ABA}$ treatment and then significantly decreased at latter time points [33]. Although we lack multiple time points of the callus development, it can be speculated that the down-regulation of ABA genes might be necessary for the callus development of Eucalyptus. Interestingly, we found seven genes encoding LEA in the callus compared to the stem (Additional file 3). However, the expression level of downregulated LEA gene (Eucgr.E00787) was much higher than the five up-regulated LEA genes. Among them, three (Eucgr.K01312, Eucgr.I01292, Eucgr.A02687) were specifically up-regulated in the callus of E. camaldulensis. The LEA genes play a pivotal role during the plant somatic embryogenesis process [34] and have been studied in some plant species like cotton [35], white spruce [36] and sweet orange [37].

In Eucalyptus, Marques identified QTLs for adventitious rooting, one QTL for sprouting ability and four QTLs for the stability of adventitious rooting [7]. Their results indicated that the phenotypic variation in these traits had a meaningful genetic component that relate to the capacity of vegetative propagation. We identified 13, 434 variants in 4723 Eucalyptus genes specific to $E$. camaldulensis (Additional file 6), including two genes encoding SERK1 and SERK2, 25 auxin related genes (e.g., auxin transporter like protein, auxin-responsive protein, auxin efflux carrier component, auxin responsive factor) and 8 genes encoding $A B A$ receptors (e.g., PYR1, PYL2, PYL4, PYL8, PYL9). Also, we found the $E$. camaldulensis specific mutated gene Eucgr.B03196 (the ARF guanine-nucleotide exchange factor GNOM, EMB30 or GN) has the potential to mediate the endosomal recycling, auxin transport and auxin-dependent plant growth [38]. Mutant EMB30 has the capacity of altering the cell wall in Arabidopsis [39]. It is also involved in the specification of apical-basal pattern formation in the early embryo and the root formation [40, 41]. Although our study lacks fundamental experiments, the pathways involved by the mutated genes in E. camaldulensis indicate that they might be associated with the SE and the regenerative ability of Eucalyptus.

TFs have been reported to function in cell division, cell growth, cell death, cell migration and organization during embryonic development, and respond to biotic and abiotic stresses. They can be another big category that affect the gene expression during the SE induction [32]. Some TFs have been reported to associate with the SE, including ABI3, WOX9-1, LEC, WUSCHEL, TALE, BBR-BPC and AP2 [32, 42]. In this study we identified some dysregulated TF genes exclusively in the callus of E. camaldulensis (Table 2, Additional file 2). Among them, ER, bHLH, MYB and WRKY TFs were upregulated. QTL analysis has identified a WRKY TF gene related to the adventitious rooting from apple hardwood cuttings [43]. In Eucalyptus, WRKY33 was up-regulated in response to the fungal affection of Chrysoporthe austroafricana [44] and Calonectria pseudoreteaudii [45]. Also, WRKY TF was up-regulated in the Eucalyptus camaldulensis seeding subjected to the water stress [46].

Jozef Šamaj discussed the structural, physiological and functional aspects connected to the role of the cell wall during embryogenesis in plant [47], including the cell wall components arabinogalactan proteins and pectins. We identified 274 and 251 DEGs related to cell wall in E. camaldulensis and E. grandis $x$ urophylla, respectively (Table 2, additional file 3). Among them, 5 and 10 genes have the capacity of encoding fasciclin-like arabinogalactan proteins and pectinesterase, respectively (additional file 3). Interestingly, Eucgr.A01158 (fasciclin-like arabinogalactan protein 11), a protein involved in the planttype secondary cell wall biogenesis, was found with upregulation in the SE of $E$. camaldulensis but downregulation in the SE of E. grandis $x$ urophylla (additional file 3). Compared to E. camaldulensis, more upregulated genes encoding pectinesterase were found in 
the SE of E. grandis $x$ urophylla (additional file 3). The functions of cell wall related genes need future experiments to be explored.

Furthermore, RP [48], HSP [49], and histone [50, 51] have been reported to function in the vegetative propagation in plants. While very few studies have been demonstrated to investigate their functions and associations in the SE of Eucalyptus, our results indicate that the dysregulation of these genes and pathways like metabolisms involved by these genes might play important roles in the SE of Eucalyptus.

\section{Conclusions}

In conclusion, we studied the transcriptome profiles during the SE of two Eucalyptus species - E. camaldulensis and E. grandis $x$ urophylla. Our results showed dysregulated genes from some gene families like auxin, ethylene, SERK, RP, ZFP, HSP, histone, ABA, LEA and TF might play key roles in the $\mathrm{SE}$ and the regenerative ability of Eucalyptus. Also, we called SNPs and small indels in $E$. camaldulensis and E. grandis $x$ urophylla, and it was revealed that genetic variants may also contribute to the $\mathrm{SE}$ and the regenerative ability of Eucalyptus. This is the first time to study the SE and the dedifferentiation in Eucalyptus using transcriptome sequencing. It will improve our understanding of the molecular mechanisms during the SE in Eucalyptus. Our findings provide a valuable resource for future studies in the field of Eucalyptus and, more importantly, will benefit the Eucalyptus breeding program.

\section{Methods}

Plants

The original seeds of E. camaldulensis (high regenerative ability, voucher ID: c0009) and E. grandis $x$ urophylla (low regenerative ability, voucher ID: j0017) were obtained from the wild in 1984 and no permissions were required to collect these plants. Then, they were confirmed by a senior botanist Prof. Dongyun Xiang and were maintained in the experimental fields of Guangxi Forestry Research Institute. The second generation of in vitro tissue-culture induced seedlings of $E$. camaldulensis and $E$. grandis $x$ urophylla were maintained on the MS medium supplemented with $20 \mathrm{mg} / \mathrm{L} \mathrm{Ca}\left(\mathrm{NO}_{3}\right)_{2}, 0.5$ $\mathrm{mg} / \mathrm{L}$ 6-BA and $0.1 \mathrm{mg} / \mathrm{L}$ IAA until 2 to $3 \mathrm{~cm}$ height. The second to the third stems from the stem tip of the seedlings were obtained and cut into $0.3 \sim 0.5 \mathrm{~cm}$ segments. About 60 segments of each Eucalyptus species were then transferred onto the induction MS medium (supplemented with $20 \mathrm{mg} / \mathrm{L} \mathrm{Ca}\left(\mathrm{NO}_{3}\right)_{2}, 1 \mathrm{mg} / \mathrm{L} \mathrm{KT}$ and $0.5 \mathrm{mg} / \mathrm{L} \mathrm{2,4-D)} \mathrm{and} \mathrm{maintained} \mathrm{in} \mathrm{dark} \mathrm{at} 28 \pm 2{ }^{\circ} \mathrm{C}$ for 10 days. Every day the callus was weighted and measured for the growth curve analysis. Stem (0 d) and complete callus $(10 \mathrm{~d})$ were used as the differentiated and dedifferentiated sample, respectively. The induction experiment was replicated three times.

\section{RNA isolation, library construction and transcriptome sequencing}

Differentiated and dedifferentiated tissues (100 mg) were collected for the total RNA isolation using the TRIzol reagent (Invitrogen) according to the manufacturer's protocol, as previously described [52, 53]. Then, the quantity and quality of the total RNA were determined by the Agilent 2100 Bioanalyzer and equal amount of total RNA $(1 \mu \mathrm{g})$ was used to construct the libraries for BGISEQ-500 RNA-Seq. Briefly, magnetic oligo (dT) beads were used to enrich the ploy(A) mRNAs, which were then fragmented into $\sim 200 \mathrm{bp}$. Then, the fragments were used for the double strand cDNA library construction by random hexamer (N6) primers, followed by the end repair using phosphate at the $5^{\prime}$ end and sticky ' $A$ ' at the 3 ' end. Next, sequencing primers were ligated to build the final cDNA library, which was then sequenced on the BGISEQ-500 RS platform at BGIShenzhen with paired-end 150 strategy.

\section{Genome mapping and gene expression profiling}

Raw data of each sample was processed by the trim_galore (https://www.bioinformatics.babraham.ac.uk/projects/trim galore/) to remove sequencing adaptors, low quality reads and contamination reads. Then, the clean data was qualitycontrolled by fastqc (http://www.bioinformatics.babraham. ac.uk/projects/fastqc/) and then aligned to the $E$. grandis genome (v2.0, https://plantgenie.org) using Hisat2 with default parameters [54]. Next, Stringtie (v1.3.4d) was used to profile gene expression in each sample [54]. TPM (transcripts per million reads mapped) method was used to normalize the gene expression for each sample. The numbers of reads aligned to Eucalyptus genes were calculated by htseq-count, as described [55].

\section{Differential expression analysis}

We used edgeR to identify differentially expressed genes in stem and callus of the two Eucalyptus species. Stringent cut-offs were employed to select the DEGs, including $\log 2$ fold change $(\log 2 \mathrm{FC})>1$ or $<-1, p$-value $<0.05$ and false discovery rate $(\mathrm{FDR})<0.1[49]$.

\section{Variant calling}

We used strelka (2.9.10-4-gd737744) to call the variants, including SNPs and small indels, in the stem and callus tissues of both E. camaldulensis and E. grandis $x$ urophylla [56]. Then, variants passed the program filter and identified in all three replicates were kept for downstream analysis. We next filtered the variants by the read depth $(>100)$ and obtained the final variants for $E$. camaldulensis and E. grandis $x$ urophylla samples. The 
variants were annotated by ANNOVAR, as previously described [55], and variants from noncoding regions and synonymous variants were discarded.

\section{Functional analysis}

To analyze the potential pathways and biological processes involved by the DEGs and mutated genes, we first annotated the E. grandis genes using the KEGG pathway and Gene Ontology databases, as previously described [53]. Then, enriched KEGG pathways and GO terms by the DEGs were identified using two statistical values: $\mathrm{p}$ value (calculated by Fisher's exact test, $<0.05$ ) and qvalue (calculated by the $R$ package 'qvalue', <0.05), as previously described [53].

\section{qRT-PCR}

We performed the quantitative real-time PCR to confirm the expression changes of candidate genes. A total of nine genes (Eucgr.A00971, Eucgr.A01091, Eucgr.B03715, Eucgr.C03048, Eucgr.D01811, Eucgr.F00490, Eucgr.F01164, Eucgr.H03077 and Eucgr.K01605) were randomly selected and the $\mathrm{H} 2 \mathrm{~B}$ gene was used as the internal control. Primers were predicted using the Primer3 and synthesized at BGIShenzhen. The procedure of qRT-PCR was same as a previous study [57]. We performed three times for each gene in every replicate $(n=9)$. After the Ct values were calculated, we used $\Delta \mathrm{Ct}$ value to present the gene expression. Then, $\Delta \Delta \mathrm{Ct}$ was used to show the difference of a gene in the callus compared to the stem. Relative normalized expression (RNE) was used to show the gene expression change: $R N E=2^{-\Delta \Delta \mathrm{Ct}}$.

\section{Supplementary Information}

The online version contains supplementary material available at https://doi. org/10.1186/s12864-020-07214-5.

Additional file 1. Gene expression profiles of differentiated and dedifferentiated tissues of E. camaldulensis and E. grandis $x$ urophylla.

Additional file 2. Differentially expressed genes in the dedifferentiated tissues compared to the differentiated tissues of $E$. camaldulensis and $E$. grandis $x$ urophylla

Additional file 3. Cell wall related DEGs identified in the SE of $E$. camaldulensis and E. grandis $x$ urophylla.

Additional file 4. Primers used for qRT-PCR.

Additional file 5. Gene variants specifically identified in E. camaldulensis.

Additional file 6. KEGG pathway enriched by the mutated genes specifically identified in E. camaldulensis.

\section{Abbreviations}

QTL: Quantitative trait loci; SERK: Somatic embryogenesis receptor kinase; HSP: Heat shock protein; TF: Transcription factor; LEA: Late embryogenesis abundant protein; TPM: Transcripts per million reads; DEG: Differentially expressed gene; GO: Gene ontology; KEGG: Kyoto Encyclopedia of Genes and Genomes; log2FC: log2 fold change; log2RNE: log2 relative normalized expression; SNP: Single nucleotide polymorphism

\section{Acknowledgements}

We acknowledge Mr. Dajie Zhou for his involvement of the transcriptome sequencing in BGI-Shenzhen.

\section{Authors' contributions}

$Y X, J L$ and $B C$ conceived and designed the experiments; $Y Z, X Z$ and $H L$ performed the experiments; $Z Q, Y X$ and $J$ analyzed the data; $Y X$ and $J$ wrote the manuscript; $B C$ revised the manuscript. All the authors have read and approved the final version of manuscript.

\section{Funding}

This work was financially supported by the Guangxi Science and Technology Program (GuiKeAD18281086), the Guangxi Forestry Science and Technology Project (2016 [11], 2014[033]), the National Natural Science Foundation of China (31400522) and the Department of Human Resources and Social Security of Guangxi Zhuang Autonomous Region (GuiCaiSheHan2018112). The authors declare that the funding bodies have no role in the research design, the data collection and analysis, and the manuscript preparation.

\section{Availability of data and materials}

The raw sequencing data can be accessed from the NCBI Sequence Read Archive (SRA) platform (http://trace.ncbi.nlm.nih.gov/Traces/sra/) under the accession number PRJNA634476.

\section{Ethics approval and consent to participate}

No specific permits were required for the described field studies. The location is not privately-owned or protected in any way, and the field studies did not involve endangered or protected species.

\section{Consent for publication}

Not applicable.

\section{Competing interests}

The authors declare that there is no conflict of interest.

Received: 27 July 2020 Accepted: 8 November 2020

Published online: 18 November 2020

\section{References}

1. Durand-Cresswell R, Boulay M, Franclet A. Vegetative Propagation of Eucalyptus. In: Bonga JM, Durzan DJ, editors. Tissue Culture in Forestry. Dordrecht: Springer Netherlands; 1982. p. 150-81.

2. Lelu-Walter M-A, Thompson D, Harvengt $L$, Sanchez L, Toribio M, Pâques LE. Somatic embryogenesis in forestry with a focus on Europe: state-of-the-art, benefits, challenges and future direction. Tree Genet Genomes. 2013;9(4): 883-99.

3. Dinkova TD, Alejandri-Ramirez ND. MicroRNA expression and regulation during plant somatic embryogenesis; 2014

4. Quan O, Li QQ, Peng HZ. PRELIMINARY REPORT ON THE DEVELOPMENT OF EMBRYOID FROM EUCALYPTUS; 1980.

5. Glória P, Clara A, Conceição S, Lucinda N. Plant regeneration by somatic embryogenesis in Eucalyptus spp.: current status and future perspectives. Southern Forests; 2013.

6. Grattapaglia D, Bertolucci FL, Sederoff RR. Genetic mapping of QTLS controlling vegetative propagation in Eucalyptus grandis and E. urophylla using a pseudo-testcross strategy and RAPD markers. Theor Appl Genet. 1995:90(7-8):933-47.

7. Marques C, Vasquez-Kool J, Carocha V, Ferreira J, O'Malley D, Liu B-H, Sederoff R. Genetic dissection of vegetative propagation traits in Eucalyptus tereticornis and E. globulus. Theor Appl Genet. 1999;99(6):936-46.

8. Thumma BR, Baltunis BS, Bell JC, Emebiri LC, Moran GF, Southerton SG. Quantitative trait locus (QTL) analysis of growth and vegetative propagation traits in Eucalyptus nitens full-sib families. Tree Genet Genomes. 2010;6(6): 877-89.

9. Ohtani M. Regulation of RNA metabolism is important for in vitro dedifferentiation of plant cells. J Plant Res. 2015;128(3):361-9.

10. Su YH, Zhao XY, Liu YB, Zhang CL, O'Neill SD, Zhang XS. Auxin-induced WUS expression is essential for embryonic stem cell renewal during somatic embryogenesis in Arabidopsis. Plant J. 2009;59(3):448-60.

11. Mantiri FR, Kurdyukov S, Lohar DP, Sharopova N, Saeed NA, Wang XD, Vandenbosch KA, Rose RJ. The transcription factor MtSERF1 of the ERF 
subfamily identified by transcriptional profiling is required for somatic embryogenesis induced by auxin plus cytokinin in Medicago truncatula. Plant Physiol. 2008;146(4):1622-36.

12. Shi $X$, Zhang $C$, Liu Q, Zhang Z, Zheng B, Bao M. De novo comparative transcriptome analysis provides new insights into sucrose induced somatic embryogenesis in camphor tree (Cinnamomum camphora L.). BMC Genomics. 2016;17:26.

13. Sato S, Toya T, Kawahara R, Whittier RF, Fukuda H, Komamine A. Isolation of a carrot gene expressed specifically during early-stage somatic embryogenesis. Plant Mol Biol. 1995;28(1):39-46.

14. Xu Z, Zhang C, Zhang X, Liu C, Wu Z, Yang Z, Zhou K, Yang X, Li F. Transcriptome profiling reveals auxin and cytokinin regulating somatic embryogenesis in different sister lines of cotton cultivar CCRI24. J Integr Plant Biol. 2013;55(7):631-42.

15. Yang $X$, Zhang $X$, Yuan D, Jin F, Zhang $Y$, Xu J. Transcript profiling reveals complex auxin signalling pathway and transcription regulation involved in dedifferentiation and redifferentiation during somatic embryogenesis in cotton. BMC Plant Biol. 2012;12:110.

16. Sharma SK, Millam S, Hedley PE, McNicol J, Bryan GJ. Molecular regulation of somatic embryogenesis in potato: an auxin led perspective. Plant Mol Biol. 2008;68(1-2):185-201.

17. Ge XX, Chai L, Liu Z, Wu XM, Deng XX, Guo WW. Transcriptional profiling of genes involved in embryogenic, non-embryogenic calluses and somatic embryogenesis of Valencia sweet orange by SSH-based microarray. Planta. 2012;236(4):1107-24.

18. Zheng Q, Perry SE. Alterations in the transcriptome of soybean in response to enhanced somatic embryogenesis promoted by orthologs of Agamouslike15 and Agamous-like18. Plant Physiol. 2014;164(3):1365-77.

19. Thibaud-Nissen F, Shealy RT, Khanna A, Vodkin LO. Clustering of microarray data reveals transcript patterns associated with somatic embryogenesis in soybean. Plant Physiol. 2003;132(1):118-36.

20. Zheng Q, Zheng Y, Perry SE. AGAMOUS-Like15 promotes somatic embryogenesis in Arabidopsis and soybean in part by the control of ethylene biosynthesis and response. Plant Physiol. 2013;161(4):2113-27.

21. Mohamed E, Claudio S, Aiming W. Molecular regulation of plant somatic embryogenesis. In: Vitro Cellular \& Developmental Biology-Plant; 2013.

22. Yang $X$, Zhang $X$. Regulation of somatic embryogenesis in higher plants. Crit Rev Plant Sci. 2010;29(1):36-57.

23. Hecht V, Vielle-Calzada JP, Hartog MV, Schmidt ED, Boutilier K, Grossniklaus U, de Vries SC. The Arabidopsis SOMATIC EMBRYOGENESIS RECEPTOR KINA SE 1 gene is expressed in developing ovules and embryos and enhances embryogenic competence in culture. Plant Physiol. 2001;127(3):803-16.

24. Cueva A, Concia L, Cella R. Molecular characterization of a Cyrtochilum loxense somatic embryogenesis receptor-like kinase (SERK) gene expressed during somatic embryogenesis. Plant Cell Rep. 2012;31(6):1129-39.

25. Hu H, Xiong L, Yang Y. Rice SERK1 gene positively regulates somatic embryogenesis of cultured cell and host defense response against fungal infection. Planta. 2005;222(1):107-17.

26. Thomas C, Meyer D, Himber C, Steinmetz A. Spatial expression of a sunflower SERK gene during induction of somatic embryogenesis and shoot organogenesis. Plant Physiol Biochem. 2004;42(1):35-42.

27. Baudino S, Hansen S, Brettschneider R, Hecht VF, Dresselhaus T, Lorz H, Dumas C, Rogowsky PM. Molecular characterisation of two novel maize LRR receptor-like kinases, which belong to the SERK gene family. Planta. 2001; 213(1):1-10

28. Maillot $P$, Lebel S, Schellenbaum $P$, Jacques A, Walter B. Differential regulation of SERK, LEC1-like and pathogenesis-related genes during indirect secondary somatic embryogenesis in grapevine. Plant Physiol Biochem. 2009;47(8):743-52.

29. Ma J, He Y, Hu Z, Xu W, Xia J, Guo C, Lin S, Cao L, Chen C, Wu C, et al. Characterization and expression analysis of ACSERK2, a somatic embryogenesis and stress resistance related gene in pineapple. Gene. 2012;500(1):115-23.

30. Singla B, Khurana JP, Khurana P. Structural characterization and expression analysis of the SERK/SERL gene family in rice (Oryza sativa). Int J Plant Genomics. 2009;2009:539402.

31. Singh P, Sinha AK. Interplay between Auxin and Cytokinin and its impact on mitogen activated protein kinase (MAPK). Methods Mol Biol. 2017;1569:93-100.

32. Mendez-Hernandez HA, Ledezma-Rodriguez M, Avilez-Montalvo RN, Juarez-Gomez YL, Skeete A, Avilez-Montalvo J, De-la-Pena C, LoyolaVargas VM. Signaling overview of plant somatic embryogenesis. Front Plant Sci. 2019;10:77.
33. Chen Z, Ji L, Wang J, Jin J, Yang X, Rao P, Gao K, Liao W, Ye M, An X. Dynamic changes in the transcriptome of Populus hopeiensis in response to abscisic acid. Sci Rep. 2017;7:42708.

34. Karami O, Aghavaisi B, Mahmoudi Pour A. Molecular aspects of somatic-toembryogenic transition in plants. J Chem Biol. 2009;2(4):177-90.

35. Roberts JK, DeSimone NA, Lingle WL, Dure L 3rd. Cellular concentrations and uniformity of cell-type accumulation of two Lea proteins in cotton embryos. Plant Cell. 1993;5(7):769-80.

36. Stasolla C, Belmonte MF, van Zyl L, Craig DL, Liu W, Yeung EC, Sederoff RR. The effect of reduced glutathione on morphology and gene expression of white spruce (Picea glauca) somatic embryos. J Exp Bot. 2004;55(397):695-709.

37. Monteiro Junior JG, Torres Dde O, da Silva MC, Ramos TM, Alves ML, Nunes Filho WJ, Damasceno EP, Brunet AF, Bittencourt MS, Pedrosa RP, et al. Nucleated red blood cells as predictors of all-cause mortality in cardiac intensive care unit patients: a prospective cohort study. PLoS One. 2015; 10(12):e0144259.

38. Geldner N, Anders N, Wolters H, Keicher J, Kornberger W, Muller P, Delbarre A, Ueda T, Nakano A, Jurgens G. The Arabidopsis GNOM ARF-GEF mediates endosomal recycling, auxin transport, and auxin-dependent plant growth. Cell. 2003;112(2):219-30.

39. Shevell DE, Kunkel T, Chua NH. Cell wall alterations in the arabidopsis emb30 mutant. Plant Cell. 2000;12(11):2047-60.

40. Richter S, Anders N, Wolters H, Beckmann H, Thomann A, Heinrich R, Schrader J, Singh MK, Geldner N, Mayer U, et al. Role of the GNOM gene in Arabidopsis apical-basal patterning--from mutant phenotype to cellular mechanism of protein action. Eur J Cell Biol. 2010;89(2-3):138-44.

41. Wolters H, Anders N, Geldner N, Gavidia R, Jurgens G. Coordination of apical and basal embryo development revealed by tissue-specific GNOM functions. Development. 2011;138(1):117-26.

42. Pinto RT, Freitas NC, Maximo WPF, Cardoso TB, Prudente DO, Paiva LV. Genome-wide analysis, transcription factor network approach and gene expression profile of $\mathrm{GH} 3$ genes over early somatic embryogenesis in Coffea spp. BMC Genomics. 2019;20(1):812.

43. Moriya S, Iwanami H, Haji T, Okada K, Yamada M, Yamamoto T, Abe K. Identification and genetic characterization of a quantitative trait locus for adventitious rooting from apple hardwood cuttings. Tree Genet Genomes. 2015;11(3):59.

44. Mangwanda R, Myburg AA, Naidoo S. Transcriptome and hormone profiling reveals Eucalyptus grandis defence responses against Chrysoporthe austroafricana. BMC Genomics. 2015;16:319.

45. Chen Q, Guo W, Feng L, Ye X, Xie W, Huang X, Liu J. Transcriptome and proteome analysis of Eucalyptus infected with Calonectria pseudoreteaudii. J Proteome. 2015;115:117-31.

46. Thumma BR, Sharma N, Southerton SG. Transcriptome sequencing of Eucalyptus camaldulensis seedlings subjected to water stress reveals functional single nucleotide polymorphisms and genes under selection. BMC Genomics. 2012;13:364.

47. Šamaj J, Bobák M, Blehová A, Pret'ová A. Importance of cytoskeleton and Cell Wall in somatic embryogenesis: Springer Berlin Heidelberg; 2005.

48. Li SW, Shi RF, Leng Y. De novo characterization of the Mung bean Transcriptome and Transcriptomic analysis of adventitious rooting in seedlings using RNA-Seq. PLoS One. 2015;10(7):e0132969.

49. Liu W, Chen M, Bai L, Zhuang Z, Fan C, Jiang N, Zhao J, Ma S, Xiang X, et al. Sci Rep. 2017;7(1):5401.

50. Uddenberg D, Valladares S, Abrahamsson M, Sundstrom JF, Sundas-Larsson A, von Arnold S. Embryogenic potential and expression of embryogenesisrelated genes in conifers are affected by treatment with a histone deacetylase inhibitor. Planta. 2011;234(3):527-39.

51. Rodrigues AS, De Vega JJ, Miguel CM. Comprehensive assembly and analysis of the transcriptome of maritime pine developing embryos. BMC Plant Biol. 2018;18(1):379.

52. Chen M, Xu R, Ji H, Greening DW, Rai A, Izumikawa K, Ishikawa H, Takahashi $\mathrm{N}$, Simpson RJ. Transcriptome and long noncoding RNA sequencing of three extracellular vesicle subtypes released from the human colon cancer LIM1863 cell line. Sci Rep. 2016;6:38397.

53. Wei S, Ma X, Pan L, Miao J, Fu J, Bai L, Zhang Z, Guan Y, Mo C, Huang H, et al. Transcriptome Analysis of Taxillusi chinensis (DC.) Danser Seeds in Response to Water Loss. PLoS One. 2017;12(1):e0169177.

54. Pertea M, Kim D, Pertea GM, Leek JT, Salzberg SL. Transcript-level expression analysis of RNA-seg experiments with HISAT, StringTie and Ballgown. Nat Protoc. 2016;11(9):1650-67. 
55. Chen M, Mithraprabhu S, Ramachandran M, Choi K, Khong T, Spencer A. Utility of circulating cell-free RNA analysis for the characterization of global Transcriptome profiles of multiple myeloma patients. Cancers. 2019:11(6):887.

56. Kim S, Scheffler K, Halpern AL, Bekritsky MA, Noh E, Kallberg M, Chen X, Kim Y, Beyter D, Krusche P, et al. Strelka2: fast and accurate calling of germline and somatic variants. Nat Methods. 2018;15(8):591-4.

57. Wei S, Wan L, He L, Wei Y, Long H, Ji X, Fu J, Pan L. De novo Transcriptome Reveals Gene Changes in the Development of the Endosperm Chalazal Haustorium in Taxillus chinensis (DC.) Danser. Biomed Res Int. 2020;2020: 7871918.

\section{Publisher's Note}

Springer Nature remains neutral with regard to jurisdictional claims in published maps and institutional affiliations.

Ready to submit your research? Choose BMC and benefit from:

- fast, convenient online submission

- thorough peer review by experienced researchers in your field

- rapid publication on acceptance

- support for research data, including large and complex data types

- gold Open Access which fosters wider collaboration and increased citations

- maximum visibility for your research: over $100 \mathrm{M}$ website views per year

At $\mathrm{BMC}$, research is always in progress.

Learn more biomedcentral.com/submissions 